\title{
GLUON PRODUCTION, COOLING AND ENTROPY IN NUCLEAR COLLISIONS
}

\author{
Jean LETESSIER ${ }^{1}$, Johann RAFELSKI ${ }^{1,2[\dagger]}$, and Ahmed TOUNSI ${ }^{1}$ \\ ${ }^{1}$ Laboratoire de Physique Théorique et Hautes Energies, Paris* \\ ${ }^{2}$ Department of Physics, University of Arizona, Tucson, AZ 85721, USA.
}

\begin{abstract}
We study the cooling (heating) of a glue-parton gas due to production (destruction) of particles and determine the associated production of entropy. We incorporate sharing of the system energy among a changing number of particles. We find that the entropy of an evolving glue-parton gas changes in an insignificant range once the initial high temperature state has been formed, despite a great change in particle number and temperature.
\end{abstract}

PACS 25.75, 5.30.C, and 12.38.M

Published in Phys. Rev. C 50 (1994) 406-409.

PAR/LPTHE/93-51

October 1993

*Unité associée au CNRS, Université PARIS 7, Tour 24, 5è ét., 2 Place Jussieu, F-75251 CEDEX 05. 
In nuclear collisions carried out at energies per nucleon considerably greater than the nucleon rest mass we observe a very high final state particle multiplicity, with which is associated an unusually large entropy content [1]. There are two questions which immediately come to mind when looking at this result: i) when and how is entropy produced in a quantum process, such as is a nuclear collision, and ii) how is the particle production related to entropy production?

We will address here the second question and investigate in a quantitative manner the increase in entropy during the evolution of the primordial gluon-parton system [2, 3, 4]. In our approach, as in every prior work up to date, we do not view the nuclear collision reaction as a reversible quantum process, but rather as a series of disorder increasing independent reactions. Thus the measurement of (conventionally defined) entropy can be performed at any instant during the collisions [3], which approach may be seen in contradiction to the prevailing understanding of reversibility of quantum evolution. This is a pragmatic approach in view of the final state formed in the collisions, which is displaying high particle multiplicity. The view we and other workers take is that a solution to the more fundamental question i) will justify the pragmatic approach taken today regarding question ii), where we proceed as if the nuclear collision reaction was a classical process involving particle production.

The physical systems of interest to us here are characterized by relatively large elastic and quasi elastic cross sections, which are typically 10-30 times larger than the particle number changing cross sections. Therefore it can be assumed that the so called kinetic equilibrium characterized by the sharing of the available energy between the particles, and the establishment of a common temperature, is reached instantaneously as compared to the slowly evolving particle number. We call a particle species to be in Hagedorn equilibrium [5], when all phase space cells are occupied according to a distribution which maximize the entropy content at some given energy. It is interesting to note here that there is so far apparently no general proof of Boltzmann's H-theorem to be found in literature for relativistic systems with particle number non-conserving reactions [6]. As expected we find below that Hagedorn equilibrium corresponds to the standard Bose/Fermi quantum distribution of particles, which give a maximum of system entropy at fixed energy. This is a preliminary step towards such a proof, which requires further that the particle numbers, to be considered in kinetic theory, evolve in time towards the Hagedorn equilibrium — while for a select system [7] this has been argued, a general discussion of this issue remains an open problem. In our detailed discussion we focus on the situation found in studies of the 'hot glue' initial state proposed by Shuryak [2]. We will show that despite a great increase in particle number as the system approaches Hagedorn equilibrium relatively little additional entropy is generated.

We begin with the considerations presented by Landau and Lifshitz [8]. The usual definition of entropy for each particle species denoted by index $l$ is:

$$
S_{\mathrm{B}, \mathrm{F}}^{l}=\int d \omega\left[ \pm\left(1 \pm n_{l}\right) \ln \left(1 \pm n_{l}\right)-n_{l} \ln n_{l}\right]
$$

where the integral is over the conventional phase space $d \omega=d^{3} p d^{3} x /(2 \pi \hbar)^{3}$ (we henceforth choose units such that $\hbar=c=1$ ). The upper sign (here + ) applies to the Bose particles ('B'), while the lower sign (here - ) applies to the Fermi particles (' $\mathrm{F}$ '). The Boltzmann limit 
follows when the occupation $n_{l}$ of the phase space by one of the particle species is small compared to unity. Given a number of particles of each species, $N^{l} \equiv \int d \omega n_{l}$, and the energy of the system $E=\sum_{l} E^{l} \equiv \int d \omega \epsilon_{l} n_{l}$, where $\epsilon_{l}=\sqrt{m_{l}^{2}+p^{2}}$, the form of $n_{l}(p, x)$ is obtained by maximizing the quantity $\mathcal{T}^{l}\left[n_{l}\right]=\mathcal{S}^{l}-\left(\alpha_{l}+\beta \epsilon_{l}\right) n_{l}$ as a functional of $n_{l}$. $\alpha^{l}$ permits to choose any given number of particles of kind $l$ and $\beta=1 / T$, which permits to keep the energy constant, is identified as the inverse temperature. Reinserting the Bose ' $\mathrm{B}$ ' and Fermi ' $\mathrm{F}$ ' quantum distribution functions

$$
n_{\mathrm{B}, \mathrm{F}}=\frac{1}{\mathrm{e}^{\beta \epsilon+\alpha} \mp 1}
$$

which follow from this consideration into the definition of entropy, Eq. (1) we obtain the explicit form 9]:

$$
S_{\mathrm{B}, \mathrm{F}}^{l}=\int d \omega\left[\frac{\beta \epsilon_{l}+\alpha_{l}}{\mathrm{e}^{\beta \epsilon_{l}+\alpha_{l}} \mp 1} \mp \ln \left(1 \mp \mathrm{e}^{-\beta \epsilon_{l}-\alpha_{l}}\right)\right]
$$

The effect of a changing particle number appears in two different ways: as a factor

$$
\gamma_{l} \equiv \mathrm{e}^{-\alpha_{l}}
$$

in front of all Boltzmann exponential present in above equations and secondly, as a coefficient of an additional additive, particle number proportional term. We can thus obtain $S_{l}$, Eq. (3), as a derivative of the partition function $\ln \mathcal{Z}^{l}\left(\beta, \alpha_{l}\right)=\mp \int d \omega \ln \left(1 \mp \mathrm{e}^{-\beta \epsilon_{l}-\alpha_{l}}\right)$.

In the Boltzmann approximation the factor $\gamma_{l}$ becomes a normalization factor which describes the average occupancy of the phase space relative to the equilibrium value [10], the additive $\alpha_{l}$-term in Eq. (3) contributes to the entropy per particle change as the occupancy changes; in particular we note that in the Boltzmann limit:

$$
\frac{S^{l}}{N^{l}}=\left.\frac{S^{l}}{N^{l}}\right|_{\mathrm{eq}}+\ln \gamma_{l}^{-1} .
$$

When we omit the last term from the discussion of the entropy of a system, we find that the entropy evolves along with the number of particles. Considering this term we see that as the occupancy of the particle phase space increases at fixed $\beta$, the entropy per particle decreases. This observation is of course not in contradiction to our expectation that the total entropy should increase as the particle number increases. The situation appears to be, however, much less trivial when we look at what happens as the occupancy of phase space approaches the equilibrium value from above $1<\gamma_{l} \rightarrow 1$. For the entropy must increase while the number of particles decreases. This can clearly only be the case if we consider how particle annihilation heats the system at given energy $E$. Similarly, we must consider when studying the increase of entropy during the approach from below to the equilibrium occupancy, how the production of particles cools the system.

We find the required change in $\beta$ for systems without conserved quantum numbers from:

$$
d E=\sum_{l} \frac{\partial E^{l}}{\partial \alpha_{l}} d \alpha_{l}+\frac{\partial E}{\partial \beta} d \beta
$$


which is inserted into the change in entropy to yield:

$$
d S=d E \frac{\partial S / \partial \beta}{\partial E / \partial \beta}+\sum_{l} d \alpha_{l}\left[\frac{\partial S^{l}}{\partial \alpha_{l}}-\frac{\partial S}{\partial \beta} \frac{\partial E^{l} / \partial \alpha_{l}}{\partial E / \partial \beta}\right] .
$$

It is our hypothesis that the system is isolated, hence $d E=0$. Considerable cancellation occurs when we evaluate Eq. (7):

$$
\left.d S^{l}\right|_{E}=d \gamma_{l} \int \frac{d \omega}{\left(1 \mp \gamma_{l} e^{-\beta \epsilon_{l}}\right)^{2}}\left[\alpha_{l} e^{-\beta \epsilon_{l}}-\epsilon_{l} e^{-\beta \epsilon_{l}} \frac{\sum_{k} \int d \bar{\omega}_{k} \alpha_{k} \epsilon_{k} \gamma_{k} e^{-\beta \epsilon_{k}}}{\sum_{k} \int d \bar{\omega}_{k} \epsilon_{k} \epsilon_{k} \gamma_{k} e^{-\beta \epsilon_{k}}}\right] .
$$

We see that indeed the change in entropy vanishes exactly when all $\alpha_{l}$ vanish, that is when $\gamma_{l} \rightarrow 1$. Furthermore we can see using Schwartz' inequality that for any $\gamma_{l}<1$ the change $d S$ is positive for positive $d \gamma_{l}$ while for $\gamma_{l}>1$ the change is negative, proving that the full Hagedorn equilibrium $\left(\gamma_{l} \rightarrow 1\right)$ gives maximum of entropy at fixed total energy.

We consider now a systems of gluons in which numerous different reactions such as $G^{*} \rightarrow G G, G G \rightarrow G G G, \ldots$, are thought to increase the number of gluons rapidly and to lead to development of Hagedorn equilibrium. Our objective is to see the usually ignored effect of cooling due to particle production, and to determine the magnitude of the associated entropy increase. Our calculations are set up such that the Hagedorn equilibrium results at $T=0.250 \mathrm{GeV}$ - due to energy conservation at fixed volume the energy density is constant as function of $\gamma_{\mathrm{G}}$ (index ' $\mathrm{G}$ ' is henceforth dropped) and takes the value $2.66 \mathrm{GeV} / \mathrm{fm}^{3}$. When we consider a thermal mass for gluons, which we take for illustrative purposes to be $m_{\mathrm{G}}^{\text {th }}=0.200 \mathrm{GeV}$, we also choose to have Hagedorn equilibrium at $T=0.250 \mathrm{GeV}$ and therefore the constant energy density drops to $1.89 \mathrm{GeV} / \mathrm{fm}^{3}$.

We show in Fig. 1 the entropy density $S / V$ (units $1 / \mathrm{fm}^{3}$ ) as function of $\gamma$. We note that for massless gluons we can not proceed beyond $\gamma=1$ because of Bose-condensation phenomena not considered further here. We show by dashed lines the same calculation carried out at $m_{\mathrm{G}}^{\text {th }}=0.200 \mathrm{GeV}$, which allows us to continue the calculation up to $\gamma \sim 2.7$. The remarkable feature to be noted in the Fig. 1 is the appearance of the very weak maximum at $\gamma=1$ - we observe that for all practical purposes in the hot glue picture [2] all entropy of the system is already created when the particle number evolution begins at $\gamma \sim 0.1$ [ [4]. However, the temperature $T$ shown in Fig. 2 evolves rapidly in the interval $0.1<\gamma<1$. While the total entropy increases by $15 \%$ (respectively $11 \%$ ) the temperature drops from $0.45 \mathrm{GeV}$ (respectively $0.43 \mathrm{GeV}$ ) to $0.250 \mathrm{GeV}$ for $m_{\mathrm{G}}^{\text {th }}=0$. (respectively $m_{\mathrm{G}}^{\text {th }}=0.2 \mathrm{GeV}$ ), this decrease being driven here solely by particle production (no expansion cooling).

As we can further note in Fig. 3 the glue number density increases considerably in the same region - it more than doubles for massless gluons. In Fig. 3 we also note that the glue number density increases monotonically as we pass $\gamma=1$ for $m_{\mathrm{G}}>0$. On the other hand, Fig. 4 shows that the entropy per particle drops continuously as could be expected from the qualitative result obtained in the Boltzmann limit, Eq. (5).

We note that in the expanding glue fireball there are no other known sources of entropy, in particular since the expansion is believed to be a largely entropy conserving hydrodynamical flow process. We will now address in qualitative terms the interplay between expansion and 


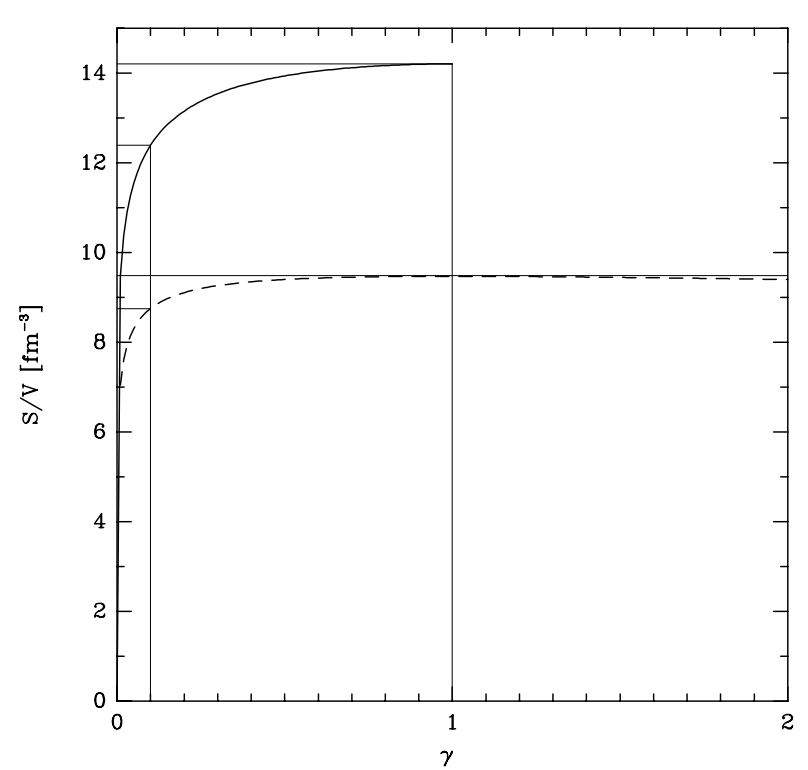

Figure 1: Entropy density $S / V$ (units $1 / \mathrm{fm}^{3}$ ) at fixed energy density $E / V=2.66 \mathrm{GeV} / \mathrm{fm}^{3}$ for $m_{\mathrm{G}}=0$ (solid line) and at $E / V=1.89$ $\mathrm{GeV} / \mathrm{fm}^{3}$ for $m_{\mathrm{G}}=0.200 \mathrm{GeV}$ (dashed line) for a (gluon) Bose gas as function of the chemical occupancy $\gamma$.

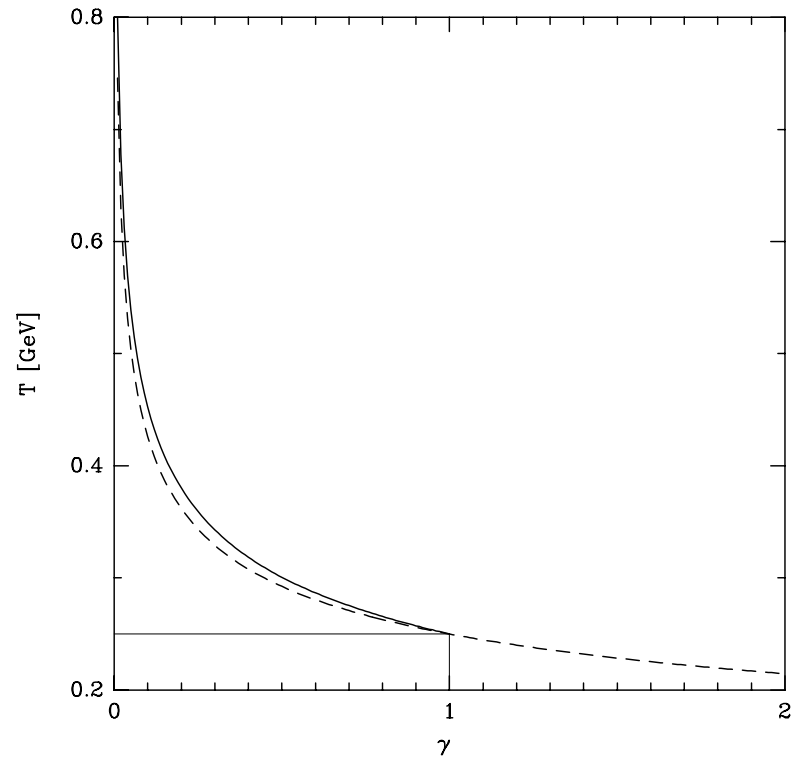

Figure 2: The temperature $T$ as function of the chemical occupancy $\gamma$. Lines as in Fig. 1; equilibrium point $\gamma=1$ has been chosen to occur at $T=0.250 \mathrm{GeV}$.

particle production cooling. In principle we need to know with some precision the relative rates of change of the volume $V$ and the occupancy factor $\gamma$. Details of the such calculations are by necessity model dependent, but we can obtain some interesting qualitative insights pursuing some general relations. For (nearly) massless gas we have:

$$
\begin{aligned}
S & =\frac{4}{3} c_{1} V T^{3} \gamma+c_{2} V T^{3} \gamma \alpha, \\
E_{\text {th }} & =E_{0}-E_{\text {flow }}=c_{1} V T^{4} \gamma .
\end{aligned}
$$

Both $c_{1}(\gamma, \beta m)$, and $c_{2}(\gamma, \beta m)$ are well known factors and can be exactly computed. For $\beta m \rightarrow 0$ we find:

$$
\frac{c_{1}}{c_{2}}=3 \frac{\sum_{n=1}^{\infty} \frac{\gamma^{n-1}}{n^{4}}}{\sum_{n=1}^{\infty} \frac{\gamma^{n-1}}{n^{3}}}=\left\{\begin{array}{r}
2.7 \text { for } \gamma=1 \\
3 \text { for } \gamma=0
\end{array}\right.
$$

In the Boltzmann limit the factors $c_{1}, c_{2}$ are independent of $\alpha=\ln \gamma^{-1}$. From Eq. (9) we obtain using $c_{1} / c_{2} \simeq 3$ :

$$
S \simeq S_{0} \frac{T_{0}}{T} \frac{1+\alpha / 4}{1+\alpha_{0} / 4}\left(1-\frac{E_{\text {flow }}}{E_{0}}\right)
$$




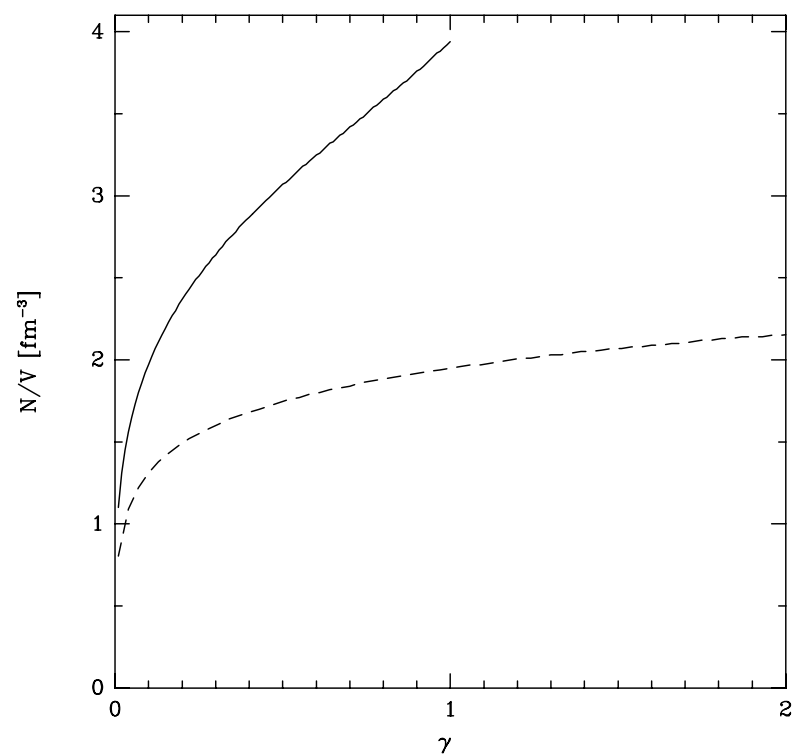

Figure 3: Particle density $N / V$ (units $1 / \mathrm{fm}^{3}$ ) as function of the chemical occupancy $\gamma$. Lines as in Fig. 1.

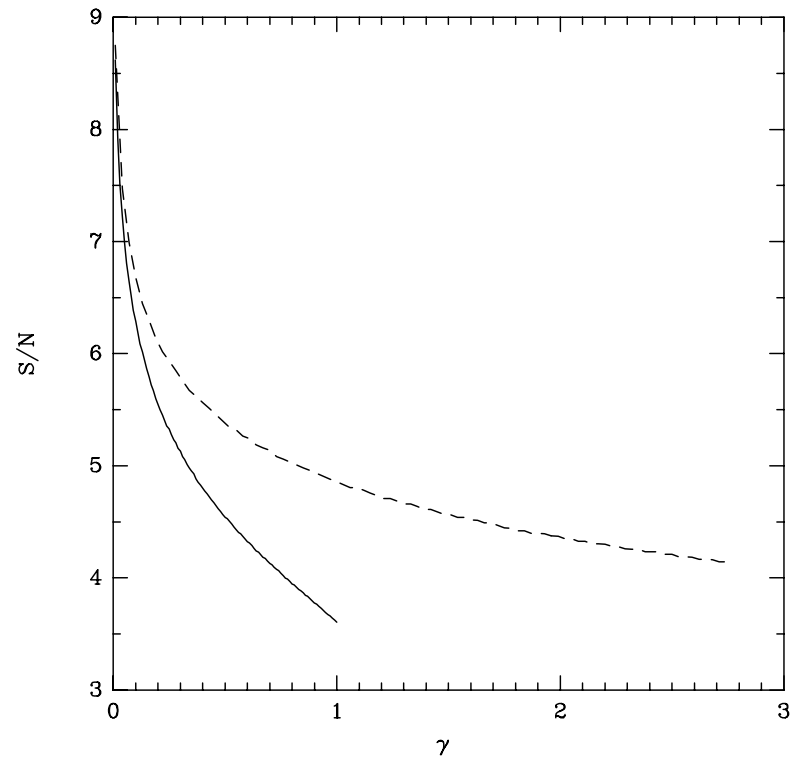

Figure 4: Entropy per particle $S / N$ for a (gluon) Bose gas as function of the chemical occupancy $\gamma$. Lines as in Fig. 1.

When $E_{\text {flow }}=0$ this is a condition satisfied by the results we presented before. Because of the appearance of the flow factor in Eq. (11) which reduces the r.h.s. we further see that a reduction of the reservoir of thermal energy entails a further reduction in the growth in entropy due to particle production.

Since entropy increase was rather small anyway, it is now possible to suppose that we have $S / S_{0} \simeq 1$. Then we find from Eq. (11) that the temperature cooling on the way to Hagedorn equilibrium is given by:

$$
\frac{T_{\mathrm{H}}}{T_{0}} \simeq \frac{1}{1+\alpha_{0} / 4}\left(1-\frac{E_{\mathrm{H}}}{E_{0}}\right)
$$

where $T_{\mathrm{H}}$ is the temperature at which the Hagedorn equilibrium is established, and there the fraction $E_{\mathrm{H}} / E_{0}$ of energy was transferred to the flow. For an initial $\gamma_{0}=0.1$ occupancy, we have $\alpha_{0}=2.3$ and hence the cooling due to particle production is by factor 0.63 (correct numerical Bose gas result which accounts for entropy production is 0.55 ). The time period is of the order of $1 \mathrm{fm} / \mathrm{c}$ and during this time the fraction of energy transferred to flow may be as large as $40 \%$. Consequently, we see that the particle production cooling (first factor in Eq. (12)) is of the same importance as the hydrodynamical expansion cooling (second factor in Eq. (12)) and that both combine to increase the temperature reduction, while both do not lead to appreciable entropy increase. We see that the cooling shown in Fig. 2 is certainly of equal if not greater importance than the cooling expected from the longitudinal hydrodynamic expansion of the glue-parton volume obtained in a recent numerical study [4].

In summary, reviewing an old topic we have found that contrary to intuitive expectations the entropy growth is not proportional to the particle number increase, and it is indeed small during approach to Hagedorn equilibrium $(\gamma \rightarrow 1)$ by a hot glue gas. We have used this 
result to show that the temperature cooling due to particle production is comparable to cooling due to hydrodynamic expansion. We have seen in detail why a sizable increase in gluon number is not generating a large increase in entropy in the nuclear collision. Our finding thus renders the mechanism of entropy production in high energy nuclear collisions even less understandable, as it now must occur in the initial (quantum) time period before $0.3 \mathrm{fm} / \mathrm{c}$ [3], when the primordial hot glue phase is generated.

Acknowledgments: We thank U. Heinz for a careful reading of the manuscript and helpful comments.

\section{References}

[†] Work supported by DOE, grant DE-FG02-92ER40733

[1] J. Letessier, A. Tounsi, U. Heinz, J. Sollfrank and J. Rafelski, Phys. Rev. Lett. 70, 3530 (1993).

[2] E.V. Shuryak, Phys. Rev. Lett. 68, 3270 (1992).

[3] K. Geiger Phys. Rev. D46, 4986 (1992).

[4] T.S. Biró, E. van Doorn, B. Müller, M.H. Thomas and X.N. Wang, Phys. Rev. C48, 1275 (1993).

[5] R. Hagedorn, Cargèse lectures in Physics, Vol. 6, Gordon and Breach, N.Y. 1973.

[6] S.R. de Groot, W.A. van Leeuwen and Ch.G. van Weert, Relativistic Kinetic Theory North Holland Pub. Co. Amsterdam 1980.

[7] S. Ochs and U. Heinz, Entropy Production by Resonance Decay and in the $\rho-\pi \pi$ system, manuscript in preparation, Regensburg 1993 and: W. Ochs, Diploma Thesis, Universität Regensburg 1993.

[8] L. Landau and E. Lifshitz, Statistical Physics, Sections 40, 54, Pergamon Press, London 1959.

[9] J. Sollfrank and U. Heinz, Physics Letters B289, 132 (1992).

[10] J. Rafelski, Phys. Lett. B262, 333 (1991) and Nucl. Phys. A544, 279c (1992). 